\title{
COMUNITAT VALENCIANA: UNA INTEGRACIÓN DE VANGUARDIA. LA ESCUELA DE ACOGIDA
}

\section{THE REGION OF VALENCIA: AN AVANT-GARDE INTEGRATION. THE SCHOOL OF RECEPTION}

\author{
Elisa María Núñez Sánchez \\ Universidad de Alicante. España/Spain \\ nunyez.eli@gmail.com
}

Recibido/Received: 17/12/2011

Modificado/Modified: 09/04/2012

Aceptado/Accepted: 30/04/2012

\section{RESUMEN}

El Programa Voluntario de Comprensión de la Sociedad Valenciana: Escuela de Acogida, único y pionero en España, está recogido en el título II del Compromiso de Integración de la Ley 15/2008 de Integración de Personas Inmigrantes en la Comunitat Valenciana (LIPIE) y con el Decreto 93/2009, por el que se aprueba el Reglamento de la citada ley. Este programa es una herramienta que voluntariamente introduce a la persona inmigrante en el conocimiento de los valores y reglas de convivencia democrática, de sus derechos y deberes, la estructura política constitucional española y los idiomas oficiales de la Comunitat; el español y el valenciano, elementos esenciales para que conozcan la sociedad que les ha acogido y se integren en ella.

\section{PALABRAS CLAVE}

Comunidad Valenciana, Inmigración, Integración, Principios básicos comunes de la Unión Europea, Escuela de Acogida.

\section{SUMARIO}

1. Radiografía de la Comunitat Valenciana. 2. Fundamentación normativa del programa escuela de acogida. 3. Contenidos del programa escuela de acogida. 4. Desarrollo e implementación del programa escuela de acogida. 5. Balance del programa voluntario de compresión de la sociedad valenciana. Bibliografía.

\begin{abstract}
The Voluntary Program of Understanding of the Valencian Society (The School of Reception), a unique and pioneer project in Spain, is included in Title II of the Commitment to Integration of Law $15 / 2008$ of Integration of Immigrant Persons in the Region of Valencia (LIPIE) and in Decree $93 / 2009$, by which the regulation of the aforementioned law is approved. This program is a tool that introduces immigrants voluntarily to the knowledge of the values and rules of democratic conviviality, their rights and duties, the politico-constitutional structure of Spain, and the official languages of the region, both Spanish and Valencian, all essential elements for said persons to know the society that has received them and in order to integrate into it.
\end{abstract}

\section{KEYWORDS}

Region of Valencia, immigration, integration, basic common principles of the EU, School of Reception. 


\section{CONTENTS}

Inside look at the Region of Valencia. 2. Regulatory basis for the School of Reception program. 3. The School of Reception program contents. 4. The development and implementation of the School of Reception. 5. Assessment of the Volunteer Program of Understanding of the Valencian Society. References.

\section{RADIOGRAFÍA DE LA COMUNITAT VALENCIANA}

La globalización ha cambiado el contexto de la migración dado que la apertura de fronteras, las nuevas tecnologías de la comunicación y el transporte más variado y barato entre otros factores, han facilitando las comunicaciones, el conocimiento y el desplazamiento de las personas, sus valores, ideas, creencias y símbolos culturales. Así, en poco más de dos décadas, España que estaba considerado a nivel europeo un país de emigración, se ha convertido en un país de inmigración alcanzando la cifra del $12,22 \%$ de población inmigrante y extranjera en su territorio en 2011. Por ello, los flujos migratorios actuales han contribuido a consolidar sociedades interculturales, enriquecidas por la pluralidad étnica, religiosa y cultural de los miembros que las conforman, cambiando en los últimos cinco años la fisonomía de la sociedad europea y de manera particular, la de la Comunitat Valenciana. Actualmente, en la Comunidad Valenciana, residen 893.759 personas inmigrantes y extranjeras, que representan más del $17,48 \%$ de la población total de nuestro territorio y que nos convierten en la segunda autonomía (sólo por detrás de Baleares) con mayor presencia de población inmigrante y extranjera en territorio español. Si sorprendente es la evolución de las personas inmigrantes y extranjeras con residencia en la Comunidad Valenciana no lo es menos su avance en el territorio español, pues el ritmo de crecimiento ha sido de medio millón de foráneos nuevos anuales.

Tabla 1. Principales nacionalidades extranjeras en la Comunidad Valenciana,

Año 2011

\begin{tabular}{|c|c|c|}
\hline Nacionalidad & Número de habitantes & Porcentaje \\
\hline Reino Unido & 144.331 & $22 \%$ \\
\hline Rumania & 143.877 & $22 \%$ \\
\hline Marruecos & 76.029 & $11 \%$ \\
\hline Ecuador & 42.864 & $6 \%$ \\
\hline Alemania & 42.481 & $6 \%$ \\
\hline Colombia & 38.731 & $6 \%$ \\
\hline Bulgaria & 37.258 & $6 \%$ \\
\hline Italia & 24.451 & $4 \%$ \\
\hline Bolivia & 22.396 & $3 \%$ \\
\hline Francia & 20.937 & $3 \%$ \\
\hline Argelia & 18.641 & $3 \%$ \\
\hline Países Bajos & 18.599 & $3 \%$ \\
\hline China & 18.282 & $3 \%$ \\
\hline Argentina & 16.565 & $100 \%$ \\
\hline TOTAL & 665.442 & 2011 \\
\hline Funte. Obs & & $2 \%$ \\
\hline
\end{tabular}

Fuente. Observatorio Valenciano de la Inmigración 2011 
Estas cifras nos muestran la realidad poblacional de la sociedad valenciana, la cual se ha transformado en un gran atanor de culturas, en la que conviven 122 nacionalidades y en la que se hablan más de 40 lenguas diferentes, entre las que cabe destacar:

Tabla 2. Principales Lenguas habladas por los extranjeros en la Comunidad Valenciana Año 2011

\begin{tabular}{|c|c|}
\hline Lengua & Porcentaje \\
\hline Ingles & $26 \%$ \\
\hline Rumano & $26 \%$ \\
\hline Español & $13 \%$ \\
\hline Árabe & $8 \%$ \\
\hline Alemán & $7 \%$ \\
\hline Búlgaro & $6 \%$ \\
\hline Italiano & $4 \%$ \\
\hline Francés & $4 \%$ \\
\hline Neerlandés & $3 \%$ \\
\hline Chino & $3 \%$ \\
\hline TOTAL & $100 \%$ \\
\hline
\end{tabular}

Fuente. Observatorio Valenciano de la Inmigración 2011

Si analizamos el fenómeno migratorio desde el punto de vista de las confesiones religiosas presentes en la Comunitat, podemos afirmar que en ella están presentes, además de católicos, evangélicos, musulmanes, mormones, ortodoxos, budistas, judíos, anglicanos e hindúes con las numerosas diversificaciones que de cada una de ellas se desprenden:

Tabla 3. Distribución de Fieles en la Comunidad Valenciana, Año 2011

\begin{tabular}{|c|c|}
\hline Religión & $\mathbf{N}^{\mathbf{0}}$ de Fieles \\
\hline Católicos & 3.801 .275 \\
\hline Otros & 642.119 \\
\hline Evangélicos & 255.080 \\
\hline Ortodoxos & 193.000 \\
\hline Musulmanes & 145.000 \\
\hline Anglicanos & 40.000 \\
\hline Budistas & 8.000 \\
\hline Mormones & 7.500 \\
\hline Judíos & 4.500 \\
\hline Hinduistas & 2.800 \\
\hline
\end{tabular}

Fuente. Observatorio Valenciano de la Inmigración 2011

Por lo tanto, de todos estos datos se deduce que ha cambiado la fisonomía de la Unión Europea en general, y de España y la Comunitat Valenciana en particular en los últimos 5 años, dada la diversidad cultural y religiosa presentes en ella, así como la convivencia de tantas y tan diferentes identidades, lo que ha llevado a la sociedad valenciana a convertirse en un pueblo diverso. Este hecho, llevó a la Unión Europea, y en especial al Gobierno Valenciano, "a plantearse un nuevo concepto de ciudadanía, un modelo innovador de integración que permitiera integrar a todos en una comunidad con una cultura política común”. Ahora bien, ¿Cómo llevarlo a cabo? ¿Cómo desarrollar modelos de integración 
que permitan no sólo la integración social de los nuevos ciudadanos en las sociedades de acogida, sino también su integración política?

\section{LA FUNDAMENTACIÓN NORMATIVA DEL PROGRAMA DE LA ESCUELA DE ACOGIDA}

Entre 1999 y 2004, el planteamiento de la Unión Europea en materia de integración de nacionales de terceros países queda claramente establecido por las conclusiones que se derivaron de las tres sesiones del Consejo Europeo:

- $\quad$ Tampere (octubre de 1999): Concluyó que era necesario un enfoque global de la inmigración que incidiera en la lucha contra la inmigración ilegal y en la promoción del retorno voluntario.

- $\quad$ Laeken (diciembre de 2001): Confirmó las conclusiones de Tampere y definió los instrumentos necesarios para integrar la política de flujos migratorios en la política exterior de la Unión Europea.

- Sevilla (junio de 2002): Consolidó el concepto de readmisión y manifestaron la disposición de la Unión para facilitar la asistencia técnica y financiera necesaria para garantizar la readmisión.

Y también por Comunicación de la Comisión al Consejo, al Parlamento Europeo, al Comité Económico y Social Europeo y al Comité de las Regiones sobre inmigración, integración y empleo de 3 de junio de 2003, la cual señaló que "Muchos Estados miembros consideran que las políticas que han aplicado hasta ahora no han sido suficientemente eficaces. Así lo demuestra la creciente preocupación ante la persistencia de obstáculos a una integración eficaz. El más frecuente es el conocimiento insuficiente de la lengua del país de acogida, pero el desempleo, un nivel de estudios insuficiente o la ausencia de cualificaciones formales se consideran también obstáculos importantes" (p. 9). Así mismo, el Consejo Europeo de Salónica de 19 y 20 de junio de 2003, subrayó la importancia de impulsar la cooperación, el intercambio de experiencias y la información sobre la integración a escala de la UE con el objeto de aprender mutuamente.

Por otro lado, el Manual sobre la integración para responsables de la formulación de políticas y profesionales, de noviembre de 2004, en respuesta al llamamiento del Consejo de Salónica, reafirmó los puntos nacionales de contacto sobre la integración y decidieron elaborar un manual de buenas prácticas. Sin embargo, determinados acontecimientos en diferentes países de Europa [4], coincidiendo con la presidencia de la Unión Europea por parte del primer Ministro Británico Tony Blair, dejaron patente que determinados modelos de integración europeos, y más concretamente el multiculturalismo británico y el asimilacionismo francés, habían fracasado; y que era necesario plantear un nuevo concepto de integración que comprenda un proceso bidireccional entre la sociedad de acogida y el recién llegado, y que no comprenda solamente el ámbito social, sino también los ámbitos cultural y político.

El punto de partida de este nuevo concepto de integración lo marcan los Principios Básicos Comunes sobre Integración aprobados por el Consejo de Ministros de Justicia y Asuntos de Interior de 19 de noviembre de 2004, los cuales señalan, entre otros, la necesidad de que "la integración sea un proceso bidireccional, entre los inmigrantes y los residentes de los Estados miembros". Así mismo señala, la importancia de adquirir por parte de los nacionales de terceros países "un conocimiento básico del idioma, la historia y 
las instituciones de la sociedad de acogida" para que así, el proceso de integración sea un éxito.

Otros de los documentos que, desde 2004, acreditan, reafirman y señalan las pautas a seguir para lograr integrar a todos los nuevos ciudadanos de la Unión europea en una comunidad política son:

Programa Común para la Integración - Marco para la Integración de los Nacionales de terceros países en la Unión Europea, Comunicación de la Comisión al Consejo, al Parlamento Europeo, al Comité Económico y Social Europeo y al Comité de las Regiones, de 1 de septiembre de 2005, que refuerza los principios básicos comunes.

Segunda edición del Manual sobre la integración para responsables de la formulación de políticas y profesionales, de mayo de 2007, que resalta “¿Qué significa la integración? Cabe esperar que la cuestión suscite debates familiares sobre la asimilación o el multiculturalismo, pero los participantes en los seminarios técnicos que preparaban el manual apenas emplearon estos términos. Como responsables políticos y profesionales que trabajaban con la integración de inmigrantes diariamente, adoptaron un enfoque mucho más práctico, centrándose en los resultados en términos de movilidad social y económica, educación, salud, vivienda, servicios sociales y participación en la sociedad. Dos procesos son críticos para mejorar la integración de los inmigrantes: la supresión de las desigualdades y la adquisición de competencias. Estos retos están en la base de las políticas de integración en Europa. [...] La adquisición de competencias, el segundo reto, se dirige a la población global, incluidos los inmigrantes, e insta a cada individuo a seguir un proceso de formación continua. La adquisición de la lengua forma parte de esta tarea, al igual que la educación y la formación continua" (Manual: 8).

Tercer Informe Anual sobre inmigración e integración de la Comisión de la Unión Europea de 11 de septiembre de 2007, en que se hace hincapié en que "la mayor parte de los Estados miembros consideran el conocimiento básico de su lengua como un elemento esencial de la integración. Muchos países centran sus estrategias de integración en programas introductorios que a veces incluyen cursos obligatorios de lengua y educación para la ciudadanía destinados a los recién llegados, y un creciente número de Estados miembros está incrementado la flexibilidad de los cursos para centrarlos en necesidades específicas; solamente algunos Estados miembros realizan una evaluación en profundidad de estas actividades." (Manual: 9).

Pacto Europeo de Inmigración y Asilo, adoptado por los 27 Estados miembros de la Unión Europea, el 16 de octubre de 2007, reafirmó la necesidad de que "las políticas de integración incluyen la educación y la formación como elementos fundamentales del proceso. Los esfuerzos de los Estados miembros se centran en clases específicas de lengua y tutorías para facilitar la integración en la escuela. Muchas iniciativas promueven el respeto de la diversidad en el ámbito educativo y el apoyo a los profesores. Sin embargo, los niños y jóvenes inmigrantes se enfrentan a retos específicos a los que deberá prestarse más atención." (Manual: 9).

Tercera Conferencia Ministerial sobre Integración. Vichy, 3 y 4 de Noviembre de 2008, considera que "la integración debe entenderse como el resultado de un proceso que brinda a los ciudadanos de terceros países que residen legalmente en un Estado miembro, la capacidad de actuar independientemente de cualquier intervención externa y de tener una condición social equiparable a la de los ciudadanos naciones y europeos". Subraya que la integración es "un proceso bidireccional que exige un compromiso mutuo y que incluye derechos y obligaciones para la sociedad de acogida y para los inmigrantes. Este proceso 
requiere una buena disposición tanto por parte de los inmigrantes a la hora de asumir la responsabilidad de integrarse en la sociedad de acogida (aprendiendo su lengua y aceptando su ordenamiento jurídico y sus valores), como de las sociedades de acogida para aceptar e integrar a los inmigrantes". Y resalta para finalizar, "que las políticas para la integración deben conllevar la aceptación plena y compartida de los valores básicos de la cultura europea, como el respeto a los derechos humanos y a la diversidad, la lucha contra la discriminación y la promoción de la igualdad de oportunidades y de la tolerancia. Además, deberán avenirse a las políticas fundamentales de la Unión Europea en materia de cohesión, empleo, desarrollo, relaciones exteriores, así como de libertad, seguridad y justicia..."

Una de las más recientes referencias a las políticas Europeas de Integración la encontramos en el 78 Pleno del Comité de las Regiones sobre los Entes Regionales y Locales en primera línea de las Políticas de Integración 12 y 13 de Febrero de 2009, el cual señala

"la necesaria participación de los entes locales y regionales en la planificación, aplicación y evaluación de las políticas de Integración, así como el papel fundamental que estos entes desempeñan a la hora de integrar a los inmigrantes en las sociedades de acogida", al tiempo que afirma que "los entes locales y regionales desempeñan un papel decisivo para la aplicación de las políticas de Integración, en función de sus competencias, en ocasiones exclusivas. Esta responsabilidad lleva consigo un considerable gasto para estas regiones y ciudades, sobre cuyo presupuesto recaen los costes de esta integración; y solicita un incremento de las asignaciones del Fondo Europeo para la Integración y un mayor apoyo a las medidas de integración a nivel local y regional”.

Por ello, el Gobierno Valenciano, a través de la aprobación de la Ley 15/2008 de Integración de Personas Inmigrantes en la Comunitat Valenciana, garantizó los derechos sociales a las personas inmigrantes residentes en la sociedad valenciana: sanidad, educación, vivienda, servicios sociales, al tiempo que en título II de la misma, el llamado Compromiso de Integración, estableció un una nueva herramienta de integración, única y pionera en España: Escuela de Acogida; un programa voluntario de comprensión de la sociedad valenciana que garantiza al nuevo valenciano, el conocimiento de los valores y reglas de convivencia democrática, los derechos y deberes, la estructura política, la cultura y los idiomas oficiales de la Comunitat Valenciana y las implicaciones de la diversidad cultural.

Este programa, apoyándose en la Ley 11/2008 de Participación Ciudadana en la Comunitat Valenciana, la cual señala en su art. 4.2. señala que "ciudadanía" es el conjunto de las personas físicas que tienen la condición política de valenciana o valenciano, de conformidad con lo dispuesto en el artículo 3 del Estatut d'Autonomia de la Comunitat Valenciana, o que, con independencia de su nacionalidad, residan en la misma, pretende además de dotar a los nuevos ciudadanos de conocimientos sobre la sociedad española en general, y de la valenciana en particular, aportarles los instrumentos necesarios para tener una plena autonomía personal que les permita hacer efectiva su ciudadanía. A nivel nacional, la trasposición de los Principios Básicos Comunes sobre Integración de la UE se realizó parcialmente tras la última reforma de la Ley Orgánica 4/2000, apoyada por el PSOE, IU y ERC. En ella, “endosa” en su art. 2. a las Comunidades Autónomas y los Municipios el deber de procurar mediante acciones formativas el conocimiento y respeto de los valores constitucionales y estatutarios de España, de los valores de la Unión Europea y el aprendizaje del conjunto de lenguas oficiales, como factores esenciales de integración de los inmigrantes 
en la sociedad de acogida. También el art. 31.7., señala que serán las Comunidades Autónomas las que acreditaran el esfuerzo de integración del inmigrante, mediante su asistencia a las acciones formativas citadas a efectos de la renovación de su situación temporal en España o la emisión del informe de arraigo.

Estas nuevas disposiciones de la LOEX sorprendieron a las Comunidades Autónomas, aunque no a la Valenciana, que con la aprobación el 5 de diciembre de 2008 el Programa Voluntario de Comprensión de la Sociedad Valenciana: Escuela de Acogida, recogido en el titulo II de la Ley de Integración de Personas Inmigrantes, ya llevaba más de una año de andadura.

\section{LOS CONTENIDOS DEL PROGRAMA DE LA ESCUELA DE ACOGIDA}

El programa voluntario de comprensión de la sociedad valenciana: Escuela de Acogida, dirigido a todo nuevo ciudadano mayor de edad, residente en la Comunitat, con 40 horas de duración, consta de 4 áreas de conocimientos, que desarrollan los siguientes contenidos:

Área de Lengua Castellana/Valenciana con 7 Bloques Temáticos:

- $\quad$ Saludos y presentaciones

- Transportes

- Compras

- Salud

- Vivienda

- El trabajo / En el trabajo

- Trámites administrativos/ Documentos

Área de Geografía de España y de la Comunitat Valenciana Geografía Física y Demográfica:

- Contextualización Social: Unión Europea, España, Comunitat Valenciana.

Área de Historia:

- Breve introducción a la Historia de la UE, España y Comunitat Valenciana anterior al siglo XX. el S. XXI.

- Evolución de la situación social y política española desde finales del S. XIX hasta

- Glosario de los acontecimientos históricos que han influido en la configuración de la Unión Europea, España y la Comunitat Valenciana

Área de Legislación Básica:

1.- Constitución Española

- $\quad$ El Estado Social y Democrático de Derecho.

- La Monarquía Parlamentaria.

- $\quad$ El Estado Autonómico.

- Valores, principios y aspectos identitarios.

- Derechos Fundamentales y Deberes Constitucionales.

- Separación de poderes y forma de gobierno parlamentaria.

- $\quad$ Los Órganos Constitucionales; La Corona; Las Cortes Generales

- $\quad$ El Gobierno; El Poder Judicial; El Tribunal Constitucional.

2.- Estatuto Autonomía Comunitat Valenciana 
- $\quad$ El modelo territorial de Estado: el Estado Autonómico. Principios.

- Organización de las Comunidades Autónomas. Sistema competencial.

- La Comunitat Autónoma Valenciana. Principios. Derechos y Deberes. Símbolos. La Generalitat.

3.- Otra Legislación Significativa

- $\quad$ Ley 4/2000 sobre Derechos y Libertades de los Extranjeros en España.

- Ley 11/2008, de la Generalitat, de Participación Ciudadana de la Comunitat Valenciana

- Ley 15/2008, de la Generalitat, de Integración de Personas Inmigrantes en la Comunitat Valenciana.

Área de Información Práctica

Temas a tratar:

- Empleo; Sanidad; Alojamiento/Vivienda

- $\quad$ Educación; Participación Ciudadana

- Trámites Administrativos

- Otras cuestiones de Interés

Al finalizar el programa, todos aquellos que hayan participado en el mismo, reciben un certificado acreditativo del Esfuerzo de Integración que pueden hacer valer no sólo en su solicitud de certificado de arraigo social en los servicios sociales, sino también en cualquiera de los siguientes supuestos:

1 - Renovación de autorización de residencia temporal no lucrativa

2 - Renovación de autorización de residencia temporal por reagrupación familiar

3 - Renovación de autorización de residencia y trabajo temporal por cuenta ajena

4 - Renovación de autorización de residencia y trabajo temporal por cuenta propia

Fueron las Universidades y Colegios Oficiales Valencianos los que supervisaron el proceso de elaboración y han avalado los textos contenidos en el manual que para tales efectos se publicó. Todo un esfuerzo colectivo que ha permitido que nuevos valencianos hayan decidido conocer y saber sobre Europa, España y la Comunitat Valenciana a través de un modelo voluntario, gratuito y bidireccional a diferencia de otros países de la UE.

\section{DESARROLLO E IMPLEMENTACIÓN DEL PROGRAMA ESCUELA DE ACOGIDA}

El programa se ha desarrollado en entidades/asociaciones y en las Agencias de Mediación y Convivencia Social - AMICS gracias a los 160 Profesores Voluntarios acreditados para impartir clases.

Así mismo desde la Dirección General de Inmigración se seleccionaron 5 profesionales cuyas funciones son:

- Revisión y adaptación pedagógica y metodológica de todos los materiales a incluir en el manual del Programa Voluntario de Comprensión de la Sociedad Valenciana, antes de su edición e impresión definitiva.

- Impartir clases en aquellas entidades y/o AMICS que no dispongan de profesorado voluntario para impartir clases de determinadas áreas de conocimiento.

- Supervisar los diferentes cursos que se realizan en las entidades y/o AMICS y realizar una evaluación de las mismas. 
Tras el primer año de implementación en forma de experiencias piloto, el Consell Valenciano aprobó la Orden por la que se regulaba la autorización para la impartición del Programa Voluntario de Comprensión de la Sociedad Valenciana: Escuela de Acogida, la Conselleria de Solidaridad y Ciudadanía, de la Generalitat debía, entre otras, autorizar a las entidades para que puedan impartir el Programa Voluntario de Comprensión de la Sociedad Valenciana Escuela de Acogida, debiendo revisar:

1. Condiciones relativas a la entidad.

2. Condiciones generales de las instalaciones.

3. Condiciones relativas al profesorado.

4. Condiciones relativas a la actividad

Lo que a su vez suponía que la Dirección General competente en materia de integración social de las personas inmigrantes debía supervisar:

a. Listado de alumnos inscritos, en el que conste el nombre y apellidos del alumnado, así como su NIE/Pasaporte, nacionalidad.

b. Hojas de firmas de control de asistencia para cada una de las sesiones del curso

c. El cuestionario de satisfacción, a cumplimentar por el alumnado de manera anónima.

d. El listado con los nombres, apellidos, número de pasaporte o NIE de las personas que han superado el curso/Programa correspondiente.

e. La relación del profesorado del que dispone la entidad en el momento del inicio de su actividad para la impartición del programa, con indicación de sus titulaciones respectivas.

f. Emisión de los certificados acreditativos del Esfuerzo de Integración.

Así mismo, se creó la Comisión de Seguimiento y Actualización del Programa Escuelas de Acogida, como órgano colegiado de seguimiento y actualización del Programa Voluntario de Comprensión de la Sociedad Valenciana Escuela de Acogida, adscrito a la Dirección General competente en materia de integración de las personas inmigrantes; y cuyas funciones son:

1. Realizar la gestión y coordinación de las actividades formativas del Programa.

2. Evaluar el grado de cumplimiento de los objetivos alcanzados con el desarrollo del Programa Voluntario de Comprensión de la Sociedad Valenciana Escuela de Acogida.

3. Concretar el modelo de indicadores a desarrollar y proponer estos indicadores para el seguimiento del cumplimiento de objetivos.

4. Proponer modificaciones puntuales para una actualización permanente del Programa Voluntario de Comprensión de la Sociedad Valenciana Escuela de Acogida.

\section{EL BALANCE DEL PROGRAMA VOLUNTARIO DE COMPRESIÓN DE LA SOCIEDAD VALENCIANA}

La implementación y desarrollo del primer curso del Programa Voluntario de Compresión de la Sociedad Valenciana: Escuela de Acogida el 14 de febrero de 2009 en la asociación Aculco-Valencia, con un total de 55 personas inscritas. Sin embargo, tal fue la acogida y el éxito del programa que a lo largo del segundo semestre de 2009 , la previsión inicial hecha desde la Dirección General de Inmigración de impartir 8 cursos piloto a lo largo de 2009 quedó obsoleta, pues el número de peticiones de asociaciones de inmigrantes que solicitaban ofrecer e impartir el programa en sus sedes aumentó, pasando de ser 4 en sus comienzos a ser 33 .

El balance global del primer año de implantación del programa fue: 
- $\quad 33$ el número de asociaciones/entidades acreditadas para impartir el Programa.

- 4 Agencias AMICS acreditadas para impartir el Programa.

- 76 cursos impartidos:

○ Provincia de Valencia: 53 en asociaciones/entidades y 1 en la Agencia AMICS de Valencia.

- Provincia de Alicante: 15 cursos en asociaciones/entidades de la provincia de Alicante y 1 en la Agencia AMICS- Orihuela.

- Provincia de Castellón: 3 cursos en asociaciones/entidades y 3 en las Agencias AMICS de Castellón y Vinaroz.

- 160 profesores voluntarios acreditados:

- 115 de asociaciones/entidades.

- 25 de Agencias AMICS.

- 13 Conselleria de Solidaridad y Ciudadanía.

- 7 FUNDAR

○ 2.600 certificados acreditativos librados:

- $\quad 1.800$ provincia de Valencia

- 600 provincia Alicante

- 200 provincia Castellón

- $\quad 3.000$ manuales editados ( $1^{\mathrm{a}}$ Edición $)$

Los 2.600 alumnos que recibieron certificado acreditativo del Programa Voluntario de Escuela de Acogida en 2009, representaban a 41 de las 122 nacionalidades diferentes que conviven en la Comunitat Valenciana y que a continuación se detallan:

Tabla 4. Nacionalidades que participan en las Escuelas de Acogida

\begin{tabular}{|l|l|l|l|l|}
\hline \multicolumn{7}{|c|}{ PAÍS } \\
\hline 1. Bolivia & 10. Colombia & 19. Argelia & 28. Chile & 37. R. Dominicana \\
\hline 2. Paraguay & 11. Ecuador & 20. Rusia & 29. Armenia & 38. R. Moldavia \\
\hline 3. Senegal & 12. Mali & 21. Costa Rica & 30. Uruguay & 39. Perú \\
\hline 4. Honduras & 13. Marruecos & 22. Gambia & 31. Gran Bretaña & 40. Mauritania \\
\hline 5. Venezuela & 14. Brasil & 23. Ucrania & 32. Camerún & 41. Ghana \\
\hline 6. Argentina & 15. Nigeria & 24. Georgia & 33. Paquistaní & \\
\hline 7. Liberia & 16. Bulgaria & 25. India & 34. Costa de Marfil & \\
\hline 8. Guinea & 17. Gambia & 26. Guatemala & 35. Rumanía & \\
\hline 9. Bielorrusia & 18. Japón & 27. Nicaragua & 36. Panamá & Nacionalidades: 41 \\
\hline
\end{tabular}

Fuente. Elaboración propia

Tabla 5. Número de Nacionalidades que participan en las Escuelas de Acogida, por pertenencia a la UUEE y Derecho de Voto

\begin{tabular}{|c|c|c|c|}
\hline & Derecho a Voto & No Derecho a Voto & TOTAL \\
\hline Comunitarios & 3 & $-\cdots$ & 3 \\
\hline No Comunitarios & 9 & 30 & 38 \\
\hline TOTAL & 12 & 30 & 41 \\
\hline
\end{tabular}

Fuente. Elaboración propia

Así mismo, el balance global del segundo año de implantación del programa fue: 
- $\quad 37$ el número de asociaciones/entidades acreditadas para impartir el Programa.

- 21 Agencias AMICS acreditadas para impartir el Programa:

$\begin{array}{cc}\circ & \text { Provincia de Valencia: } 12 \\ \circ & \text { Provincia de Alicante: } 5 \\ \circ & \text { Provincia de Castellón: } 4 \\ \text { - } & \text { 224 cursos impartidos } \\ \circ & \text { Provincia de Valencia: } \\ \circ & 145 \text { en asociaciones/entidades. } \\ \circ & 17 \text { en Agencias AMICS. } \\ \circ & \text { Provincia de Alicante: } \\ \circ & 32 \text { en asociaciones/entidades de } \\ \circ & 8 \text { en Agencias AMICS. } \\ \circ & \text { Provincia de Castellón: } \\ \circ & 17 \text { en asociaciones/entidades. } \\ \circ & 5 \text { en Agencias AMICS. } \\ \text { - } & 7.000 \text { certificados librados: } \\ \circ & 4.850 \text { provincia de Valencia } \\ \circ & 1.600 \text { provincia Alicante } \\ - & 550 \text { provincia Castellón } \\ \text { - } & 7.000 \text { manuales (2 } 2^{\text {a }} \text { Edición) }\end{array}$

Tabla 6. Cuadro resumen resultados Escuelas de Acogida - Por Provincias

\begin{tabular}{|c|c|c|c|c|c|c|}
\hline & Entidades & Cursos entidades & AMICS & Cursos AMICS & Total cursos & Diplomas \\
\hline Valencia & 24 & 145 & 12 & 17 & 142 & 4.850 \\
\hline Castellón & 3 & 17 & 4 & 5 & 12 & 550 \\
\hline Alicante & 7 & 32 & 5 & 8 & 33 & 1.600 \\
\hline Total & 37 & 194 & 21 & 30 & 224 & 7.000 \\
\hline
\end{tabular}

Fuente: Elaboración Propia

\section{CONCLUSIÓN}

A la vista de los resultados obtenidos, puedo afirmar que Escuela de Acogida es un instrumento de integración en plena consonancia con las medidas sobre integración adoptadas en la Unión Europea, una herramienta que voluntariamente introduce a la persona inmigrante en el conocimiento de los valores y reglas de convivencia democrática, de sus derechos y deberes, de la estructura política constitucional española y los idiomas oficiales de la Comunitat; el español y el valenciano, elementos esenciales para que conozcan la sociedad que les ha acogido y se integren en ella.

\section{BIBLIOGRAFÍA}

CÁTALA i BAS, A., (2009), "La integración de la inmigración en la Comunidad Valenciana" en Revista General de Derecho Público Comparado, no 5 (2009), Madrid, Iustel, pp. 1-25

FELIP i SARDÀ, J.M.; NÚÑEZ SÁNCHEZ, E.M. y ORTEGA ROIG, M. (2009), "Comunitat Valenciana. El modelo valenciano de integración de los nuevos ciudadanos" en TUR AUSINA, R. 
(coord.), La integración de la población inmigrante en el marco europeo, estatal y autonómico español. Madrid: Iustel, pp. 321-349.

GUIRAUDON, V. (2008), Contratos de integración para inmigrantes: tendencias comunes y diferencias en la experiencia europea, Área: Demografía, Población y Migraciones Internacionales, ARI núm. 43/2008. Madrid: Real Instituto Elcano.

MICHALOWSKI, I. (2007), "Modelos de acogida en Alemania, Francia y los Países Bajos: diseño y efectividad de los programas de acogida e integración", en Biles, J; Michalowski, I. y Winnemore, L. (2007), Políticas y modelos de acogida. Una mirada transatlántica. Canadá, Alemania, Francia y los Países Bajos, Serie Migraciones, nº. 12. Barcelona: Fundación CIBOB, pp. 67-92.

SOLANES CORELLA, A. (2009), “¿Integrando por ley?: de los contratos europeos de integración al compromiso de la Ley autonómica valenciana 15/2008" en Revista de derecho migratorio y extranjería, $\mathrm{n}^{\circ} .20$, pp. 47-75.

VV.AA. (2009), Manual Escuelas de Acogida. Valencia: Generalitat Valenciana.

\section{Breve currículo:}

\section{Elisa María Núñez Sánchez.}

Licenciada en Ciencias Religiosas por la Universidad Pontificia de Comillas de Madrid. Experta universitaria en Educación Sociopolítica por la Universidad Católica de Valencia. En la actualidad está elaborando la tesis sobre las políticas de integración de las personas inmigrantes y extranjeras en la Comunitat Valenciana en la Universidad de Alicante. Su formación se completa con estudios y seminarios sobre diferentes aspectos del fenómeno migratorio actual y su integración en las sociedades de acogida. Ha sido coordinadora del libro-manual Escuela de Acogida: Programa Voluntario de Comprensión de la Sociedad Valenciana. 